\title{
Stoney formula: Investigation of curvature measurements by optical profilometer
}

\author{
Maria Rosa Ardigo ${ }^{1, a^{*}}$, Maher Ahmed ${ }^{1, b}$ and Aurélien Besnard ${ }^{1, c}$ \\ ${ }^{1}$ Arts et Metiers ParisTech - LaBoMaP, Rue Porte de Paris, 71250 Cluny, France \\ amaria-rosa.ardigo@ensam.eu, bmaher.ahmed@ensam.eu, caurelien.besnard@ensam.eu
}

Keywords: Thin films, Stoney formula, substrate curvature, optical profilometer

\begin{abstract}
Thin films' residual stress is often determined by the Stoney formula, using the measurements of the substrate curvature, even if the required hypotheses are not completely respected. In this study, a $2.2 \mu \mathrm{m}$ titanium nitride coating was deposited by reactive sputtering on a silicon substrate. The Stoney formula was used in order to calculate the residual stress of the film. The radius of curvature was measured, before and after coating by optical profilometer, considering the whole surface of the sample. The effect of the substrate shape (square and rectangular) with various dimensions was investigated. We showed that the shape of the substrate influence strongly the deformation. Moreover, it was highlighted that the choice of the radius (maximum value, minimum value, mean value, with or without initial curvature correction) is critical to the determination of the stress.
\end{abstract}

\section{Introduction}

From a qualitatively point of view, thin films' total stress can be written as follow:

$\sigma_{\text {tot }}=\sigma_{\text {ext }}+\sigma_{\text {th }}+\sigma_{\mathrm{i}}$

where $\sigma_{\text {ext }}$ represents the external stress due to the external loading, $\sigma_{\mathrm{th}}$ the thermally-induced stress and $\sigma_{\mathrm{i}}$ the internal stress. The thermal component can be estimated using the following equation:

$$
\sigma_{\text {th }}=\mathrm{E}_{\mathrm{f}}\left(\alpha_{\mathrm{f}}-\alpha_{\mathrm{s}}\right)\left(\mathrm{T}_{\mathrm{S}}-\mathrm{T}_{\mathrm{M}}\right)
$$

with $\mathrm{T}_{\mathrm{S}}$ the coating temperature, $\mathrm{T}_{\mathrm{M}}$ the temperature at which the thermal stress are determined, or at which the coating system operates in service, $E_{\mathrm{f}}$ the Young modulus of the film, $\alpha_{\mathrm{f}}$ and $\alpha_{\mathrm{s}}$ the film and the substrate thermal expansion coefficients. In the case of our study $\sigma_{\text {ext }}$ is equal to zero, because no external load is applied. Moreover, in our experimental conditions and using for $\mathrm{E}_{\mathrm{f}}, \alpha_{\mathrm{f}}$ and $\alpha_{\mathrm{s}}$ the values reported in the literature $[1,2]$, we obtain for $\sigma_{\text {th }}$ a negligible value (between 10 and $-30 \mathrm{MPa}$ ). For these reasons, only the internal stress were considered and evaluated.

The Stoney formula is generally used to calculate the residual stress value [3]:

$$
\sigma=\frac{E_{s}}{6\left(1-v_{s}\right)} \frac{h_{s}^{2}}{h_{f}}\left(\frac{1}{R}-\frac{1}{R_{0}}\right)
$$

with $E_{s}$ the Young's modulus, $v_{s}$ the Poisson's ratio and $h_{s}$ the thickness of the substrate, $h_{f}$ the thickness of the film, $\mathrm{R}$ and $\mathrm{R}_{0}$ the curvature radii of the substrate after and before deposition.

However, the Stoney formula requires the respect of the following hypotheses $[4,5]$ :

- the thicknesses of the substrate and the coating are smaller than the lateral dimensions;

- deformations and rotations are infinitesimal;

- the thickness of the coating is smaller than the thickness of the substrate;

- the substrate and the coating are homogenous, isotropic and linear elastic;

- the radius of curvature is equal in all directions (spherical deformation);

- the stress and the radius of curvature are constant on the whole surface of the plate.

Nevertheless, the Stoney formula is frequently used, even if these hypotheses are not completely respected [6]. 
Concerning the determination of the radius of the curvature, several methods are reported in the literature. For instance, Masters and Salamon [7] and Harper and $\mathrm{Wu}$ [8] used punctual curvatures measured in the center and at a quarter of the sample. Ngo et al. [8] showed that the film stress depends on curvatures of the entire substrate and non-locally on the substrate curvature. Consequently, they extended the Stoney formula for non-uniform misfit strain distribution. Another different approach was used by Fillon [9]. The hypothesis of the uniformity of the curvature was assumed, and the stress evolution in the film was monitored in real time during growth, using a multi-beam optical stress sensor technique. The substrate curvature was measured for two orthogonal directions. For each direction, six measurements were realized and the average value was considered.

In the present study a TiN thin film was deposited on silicon substrates with different size and shape on the same time. The substrate curvature was measured, before and after coating, taking into account the whole surface. The residual stress was determined using the Stoney formula adapted to a plate-like substrate (Eq. 3). We showed that the deformation is influenced by the shape of the substrate. Moreover, even if it is well known that the substrate curvature before coating has to be precisely measured, since the substrate flatness is never perfect, the literature never define which radius has to be used for the calculus (maximum, minimum or mean value). In the present work we put in evidence that the choice of the radius is critical for the determination of the stress.

\section{Experimental conditions}

A TiN coating was deposited on a $380 \mu \mathrm{m}$ (001) silicon substrate by dc reactive magnetron sputtering, using a titanium metallic target (purity 99.99\%) in an $\mathrm{Ar}+\mathrm{N}_{2}$ gas mixture. The working pressure was $0.65 \mathrm{~Pa}$ and the initial substrate temperature was at $55^{\circ} \mathrm{C}$. The discharge potential was $370 \mathrm{~V}$ and the substrate was at the floating potential, which was measured during process around $18 \mathrm{~V}$. The $\mathrm{Ar}$ and $\mathrm{N}_{2}$ flow rate were $90 \mathrm{sccm}$ and $40 \mathrm{sccm}$ respectively. In these conditions the growing rate of the TiN film was at the order of $83 \mathrm{~nm} \cdot \mathrm{min}^{-1}$. The deposition time was adjusted in order to obtain a film with a constant thickness close to $2.2 \mu \mathrm{m}$.

Before the deposition process, the Si substrates are cut from a one side mirror-polished wafer, cleaned in an ultrasonic bath, first with acetone and then ethanol solutions, dried and kept at room temperature. The TiN film was deposited on the mirror-polished surface. In order to investigate the effect of the shape of the substrate on the deformations, five samples were tested with different size and shape, from square $(10 \times 10$ and $15 \times 15 \mathrm{~mm} \times \mathrm{mm})$ to rectangular $(10 \times 13,10 \times 15,10 \times 19$ $\mathrm{mm} \times \mathrm{mm}$ ). The curvature radius was measured by an optical profilometer (WYKO NT1100) for both sides of the sample (mirror polished and unpolished) before and after TiN deposition, considering the whole surface of the sample. The values and the directions of the radii of curvature were obtained by Gwyddion ${ }^{1}$, which determine the curvature of a surface with two orthogonal directions. The residual stress was then calculated using the modified Stoney formula for a platelike substrate (Eq. 3).

In this study we will focus only on the results concerning the mirror polished side.

\section{Results and discussion}

\section{Influence of the shape of the samples on the deformations.}

In the Table 1 are listed the values of the radii of curvature measured for the five samples for two orthogonal directions of the surface $\left(\varphi_{1}\right.$ and $\left.\varphi_{2}\right)$, before and after coating $\left(R_{0.1}, R_{0.2}\right.$ and $R_{1}, R_{2}$ respectively):

\footnotetext{
${ }^{1}$ http://gwyddion.net/
} 
Table 1: Radii of curvature measured for the investigated samples.

\begin{tabular}{|c|c|c|c|c|}
\hline Sample number & $\mathbf{R}_{\mathbf{0 . 1}}(\mathbf{m})$ & $\mathbf{R}_{\mathbf{0 . 2}}(\mathbf{m})$ & $\mathbf{R}_{\mathbf{1}} \mathbf{( m )}$ & $\mathbf{R}_{\mathbf{2}}(\mathbf{m})$ \\
\hline 1 & -47.2 & -94.4 & 67.2 & 12.7 \\
\hline 2 & 57.5 & 13.6 & 10.1 & 8.1 \\
\hline 3 & 127.7 & 35.2 & 17.8 & 8.4 \\
\hline 4 & -294.2 & 39.5 & 21.9 & 9.4 \\
\hline 5 & -32.8 & 58.8 & 26.7 & 10.9 \\
\hline
\end{tabular}

In a first time we chose to neglect the initial curvature correction of the substrate and to consider only the radii measured after coating $\left(\mathrm{R}_{1}\right.$ and $\left.\mathrm{R}_{2}\right)$. For the sample 1 an important difference (about $80 \%$ ) can be observed between $\mathrm{R}_{1}$ and $\mathrm{R}_{2}$ values, which correspond to an elliptical deformation (Fig. 1a). On the contrary, for the sample 2, the values obtained for $\mathrm{R}_{1}$ and $\mathrm{R}_{2}$ suggest that, after coating, the sample tends to a spherical deformed shape (Fig. 1b). The difference between $R_{1}$ and $R_{2}$ values is nearly $20 \%$.

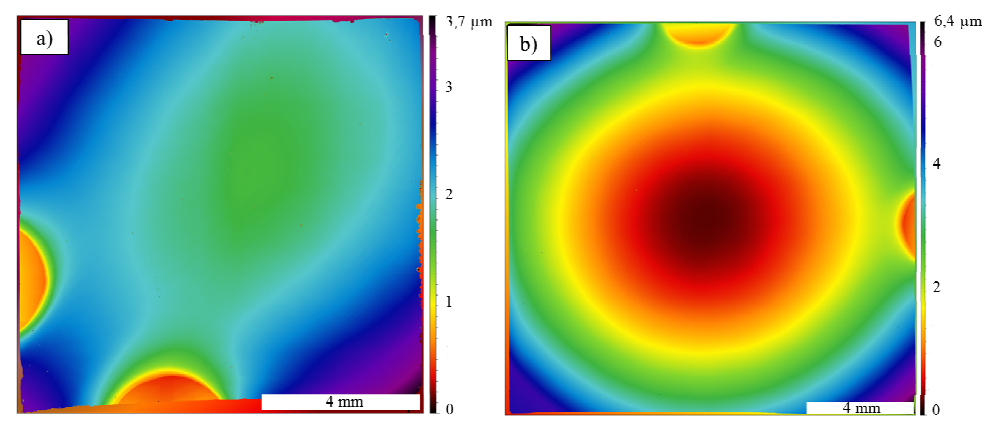

Figure 1: Deformation after coating on sample 1 (a) and 2 (b).

Concerning the rectangular samples (samples 3, 4 and 5), in all cases after coating we observed an elliptical deformation. The difference between $\mathrm{R}_{1}$ and $\mathrm{R}_{2}$ values is equal to $53 \%$ for the sample $3,57 \%$ for the sample 4 and $59 \%$ for the sample 5 . These results suggest that the nature of the deformations after coating doesn't depend on the geometry and on the size of the samples.

The results presented in table 1 show clearly that the initial substrate is not flat and that the initial deformation cannot be neglected for the stress calculation. In this aim, we determined $\Delta_{1}=\frac{1}{\mathrm{R}_{1}}-\frac{1}{\mathrm{R}_{0.1}}$ and $\Delta_{2}=\frac{1}{\mathrm{R}_{2}}-\frac{1}{\mathrm{R}_{0.2}}$ and we use for convenient the equivalent radii $\mathrm{R}_{\text {lequivalent }}=\Delta_{1}^{-1}$ and $R_{2 \text { equivalent }}=\Delta_{2}^{-1}$. The percent of difference between $R_{1 \text { eq }}$ and $R_{2 \text { eq }}$ is shown in the figure 2, with those between $\mathrm{R}_{1}$ and $\mathrm{R}_{2}$ for comparison.

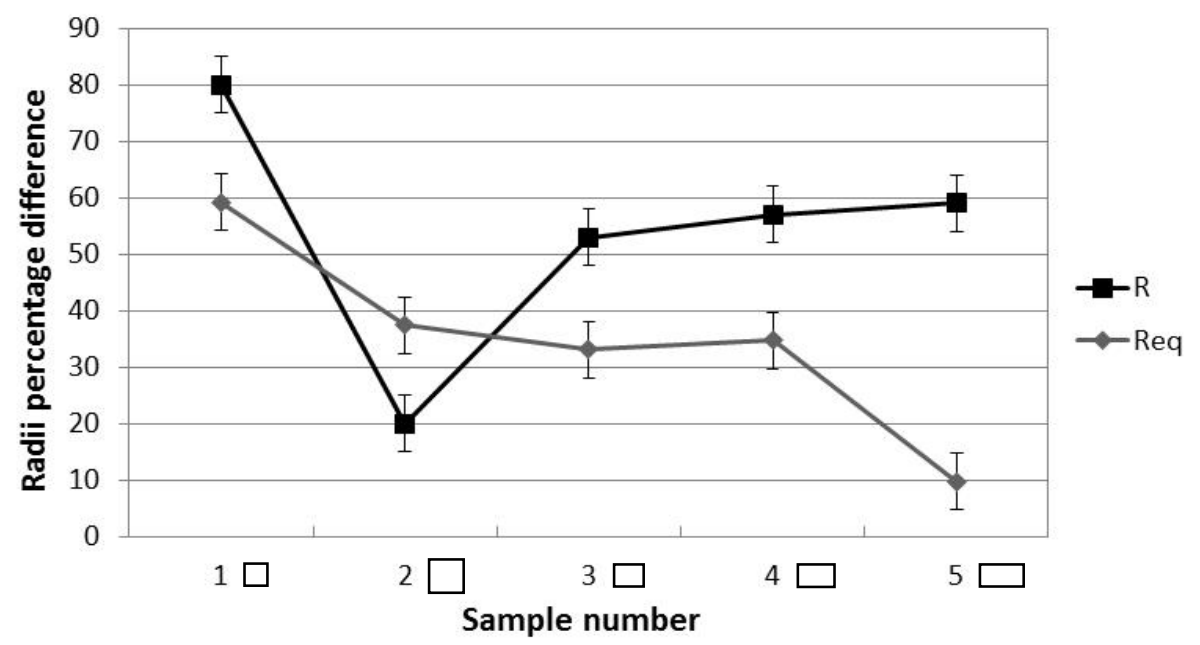

Figure 2: Percentage difference between $R_{\text {eq1 }}$ and $R_{\text {eq2 }}$ values and $R_{1}$ and $R_{2}$ values. 
If the percentage difference of the radii after coating without initial curvature correction doesn't show any particular trend, it is not the case with this correction. A size effect is detected for square substrate and then a shape effect. This means that for a sufficient size, when the shape of the sample approaches to a strip, the deformation after coating tends to become more spherical. These observations are in agreement with the works of Guyot et al. [8].

Gwyddion allows obtaining not only the values of the curvature radii before and after coating along the principal perpendicular directions of the surface, but also the values of the curvature directions $\left(\varphi_{1}\right.$ and $\left.\varphi_{2}\right)$. As they are orthogonal, only $\varphi_{1}$ variation is considered. The measured values are shown in the figure 3 .

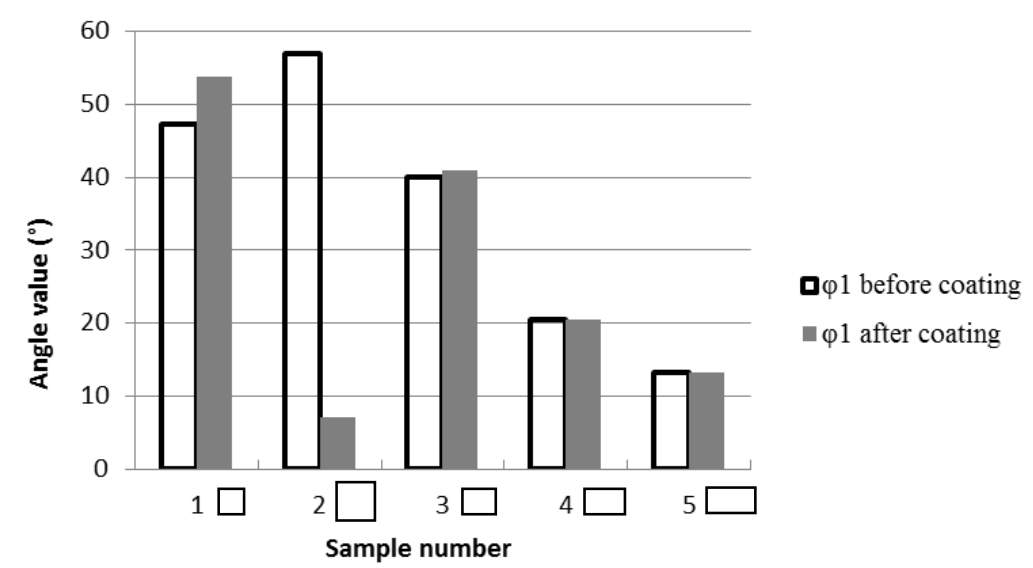

Figure 3: Variation of $\varphi_{1}$ before and after coating. $\varphi_{2}=\varphi_{1}[\Pi / 2]$.

The comparison of the results obtained for the five samples shows that, before and after coating, the curvature directions are very similar $\left( \pm 5^{\circ}\right)$, with the only exception of the sample $2(15 \times 15$ $\mathrm{mm})$, with a difference about $45^{\circ}$. This suggests that the initial orientation of the substrate influence the orientation after coating.

\section{Residual stress values determination.}

The residual stress was determined using the modified Stoney formula for a plate-like substrate (Eq. 3). Positive signs of the curvature radii correspond to a concave surface, whereas negative signs to a convex one; mixed signs mean a saddle-like surface. We used the values of $130 \mathrm{GPa}$ for the substrate Young's modulus $\left(\mathrm{E}_{\mathrm{s}}\right)$ and 0.28 for the substrate Poisson's ratio $\left(v_{\mathrm{s}}\right)[10,11]$. The thickness of the substrate $\left(h_{\mathrm{s}}\right)$ is given at $386 \pm 3.5$ by the suppliers and the thicknesses of the films $\left(\mathrm{h}_{\mathrm{f}}\right)$ were measured by high resolution scanning electron microscopy with a magnification of 25000 and are equal to $2.2 \pm 0.1 \mu \mathrm{m}$. $\mathrm{R}$ and $\mathrm{R}_{0}$ are the curvature radii of the substrate after and before coating and their values are listed in the table 1.

The residual stress was calculated using the final radii $\mathrm{R}$, where the initial curvature of the substrate is neglected, and the equivalent radii $R_{\text {eq }}$, where it is taken into account.

As the radii of curvature were measured along the principal orthogonal directions of the surface, we calculated, for each of the five samples, the maximum, the minimum and the mean value of $R$ and $\mathrm{R}_{\text {eq. }}$. The highest radii will correspond to a smallest stress, and the smallest radii to the highest stress. The mean value is the most used in the literature. From that, we could calculate three different values of the residual stress for each sample. Of course because we used substrate from the same wafer, and that the coatings were deposited on the same time with a large target (16" 5 "), which guarantees a homogeneous deposition zone, we should obtain exactly the same stress in all cases. But, with the radii measurements presented previously, it is clear that there will be differences in the measured stress with the change of substrate shape and the choice of the radii $\left(\mathrm{R}\right.$ or $\left.\mathrm{R}_{\mathrm{eq}}\right)$. The results are shown in the figure 4. 

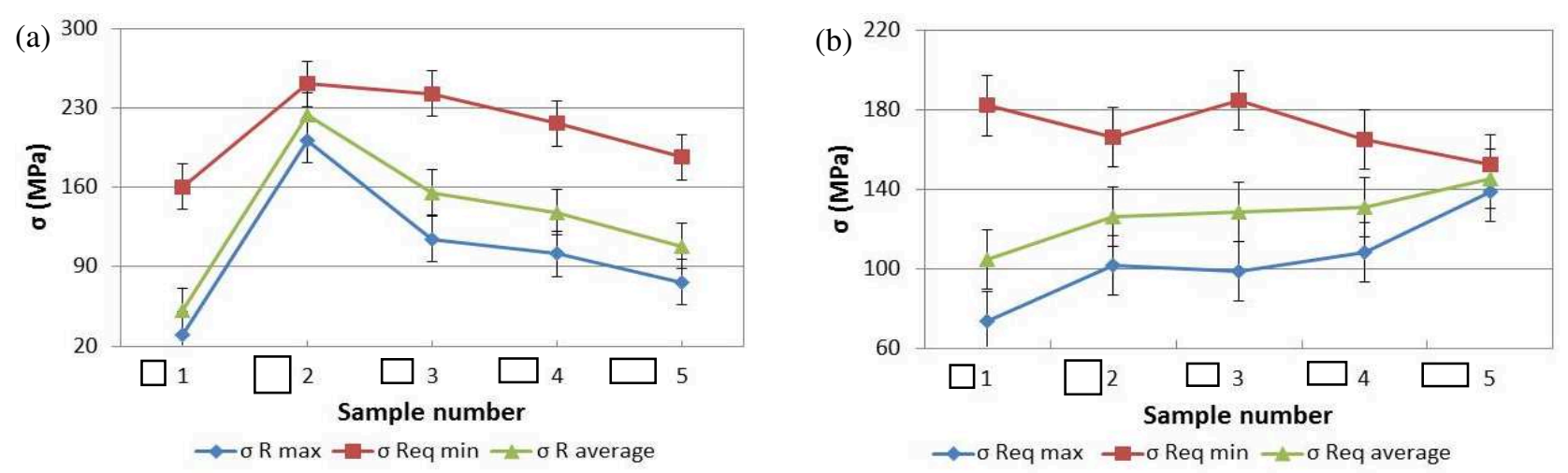

Figure 4: Residual stress calculated using $R(a)$ and $R_{e q}(b)$ values.

These graphs show clearly the importance of the initial curvature. In the case without, the stress takes values from 30 to $250 \mathrm{MPa}$, and there is no trend with the change of shape of the substrate. In the case with the initial curvature, the stress takes values from 70 to $190 \mathrm{MPa}$, and there is a clear "shape effect". More the substrate is strip-like, with probably a minimum size, more the stress is homogeneous and Stoney's hypothesis validated. Second conclusion, if there were no shape effect, we could assumed a mean value for the five samples and so compare the influence of the choice of the radius used for the stress calculation. The stress calculated in the first case takes values of $105 \pm 65,135 \pm 65$ and $210 \pm 40 \mathrm{MPa}$ for $\mathrm{R}_{\max }, \mathrm{R}_{\text {average }}$ and $\mathrm{R}_{\min }$, respectively. In this case, it cannot be determined with precision, as the maximal variation could be around $200 \mathrm{MPa}$, more or equal to the mean value calculated. In the second case, the stress takes values of $105 \pm 25,130 \pm 15$ and $170 \pm 15$

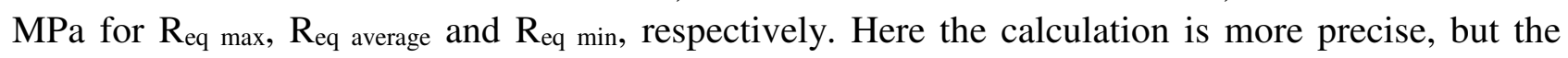
maximal variation is about $105 \mathrm{MPa}$, in the order of the smallest stress mean value. Because of the low stress level it is not so easy to conclude on the choice of the radius. But, even both equivalent radii (min and max) should be used, we could assume that the minimum equivalent radius, corresponding to the highest deformation of the substrate, gives more precise results.

The films studied in this paper present low stress level, in the order of a few hundred of MPa. These results are in agreement with the work of Bielawski [12], who studied the residual stress variation in a TiN/Si coating as a function of the bias voltage and of the deposition pressure. Indeed, in working conditions similar to our experimental parameters, they found tensile residual stress values included between 0 and $200 \mathrm{MPa}$. Nevertheless, this level is lower than those found by some other authors for TiN films [1, 13, 14, 15]. This difference may be partially explained by different coating conditions (pressure, temperature, bias, etc.), substrate thicknesses and values used for the Young's modulus and/or the Poisson's coefficient of the substrate. They ( $E_{\mathrm{s}}$ and $\left.v_{\mathrm{s}}\right)$ depend on the substrate direction and cover a wide range of values $[10,11]$. Another possible explanation may be the presence on the unpolished side of our silicon substrates of high residual stress before coating (293 MPa calculated with the X-ray diffraction technique, using the $\sin ^{2} \psi$ method [16]), when the polished one is at $90 \mathrm{MPa}$. It might be considered as an external load, and. As a consequence, the initial hypotheses ( $\sigma_{\text {ext }}$ equal to zero) should be modified.

\section{Conclusions}

The results obtained in our study show that the shape of the substrate before coating has an influence on the deformations after coating. Indeed, it was put in evidence that, when the shape of the sample approaches a strip, the deformation after coating tends to become more spherical. This result is in agreement with the available literature data. Moreover, it was clearly shown that the choice of the curvature radius (maximum, minimum or mean value) is a critical parameter for the determination of the stress. A careful attention should be given to this technique to minimise the dispersion of the calculated stress values and to ensure the relevance of the studies. The stress value is an image of the curvature radius and, as a consequence, of the way it was determined. 


\section{References}

[1] F. Vaz, L. Rebouta, Ph. Goudeau, J.P. Rivière, E. Schäffer, G. Kleer, M. Bodmann, Residual stress states in sputtered $\mathrm{Ti}_{1-\mathrm{x}} \mathrm{Si}_{\mathrm{x}} \mathrm{N}_{\mathrm{y}}$ films, Thin Solid Films 402 (2002) 195-202.

[2] G. Dehm, D. Weiss, E. Arzt, In situ transmission electron microscopy study of thermal-stressinduced dislocations in a thin $\mathrm{Cu}$ film constrained by a Si substrate, Mat. Sci. Eng. A309-310 (2001) 468-472.

[3] G.G. Stoney, The tensions of metallic films deposited by electrolysis, Proc. R. Soc. Land. A82 (1909) 172-175.

[4] N. Guyot, Y. Harmand, A. Mézin, The role of the sample shape and size on the internal stress induced curvature of thin-film substrate systems, Int. J. Sol. Struct. 41 (2004) 5143-5154.

[5] A. Mézin, Coating internal stress measurement through the curvature method: a geometry based criterion delimiting the relevance of Stoney's formula, Surf. Coat. Technol. 200 (2006) 5259-5267.

[6] D. Ngo, X. Feng, Y. Huang, A.J. Rosakis, M.A. Brown, Thin film/substrate systems featuring arbitrary film thickness and misfit strain distributions. Part I: Analysis for obtaining film stress from non-local curvature information, Int. J. Sol. Struct. 44 (2007) 1745-1754.

[7] C.B. Masters, N.J. Salamon, Geometrically nonlinear stress-deflection relations for thin film/substrate systems with a finite element comparison, ASME J. Appl. Mech. 61 (1994) 872-878.

[8] B.D Harper, C.-P. Whu, A geometrically nonlinear model for predicting the intrinsic film stress by the bending-plate method, Int. J. Solid Structure 26 (5-6) (2009) 511-525.

[9] A. Fillon, G. Abadias, A. Michel, C. Jaouen, Stress and microstructure evolution during growth of magnetron-sputtered low-mobility metal films: Influence of the nucleation conditions, Thin Solid Films 519 (2010) 1655-1661.

[10] J.J. Wortman, R.A. Evans, Young's Modulus, Shear Modulus and Poisson's Ratio in Silicon and Germanium, J. Appl. Phys. 36 (1) (1965) 153-156.

[11]D.R. França, A. Blouin, All-optical measurement of in-plane and out-of-plane Young's modulus and Poisson's ratio in silicon wafers by means of vibration modes, Meas. Sci. Technol. 15 (2004) 859-868

[12]M. Bielawski, Residual stress control in TiN/Si coatings deposited by unbalanced magnetron sputtering, Surf. Coat. Technol. 200 (2006) 3987-3995.

[13]L. Zhang, H. Yang, X. Pang, K. Gao, A. A. Volinsky, Microstructure, residual stress, and fracture of sputtered TiN films, Surf. Coat. Technol. 224 (2013) 120-125.

[14]F. Vaz, P. Cerqueira, L. Rebouta, S.M.C. Nascimento, E. Alves, Ph. Goudeau, J.P. Rivière, K. Pischow, J. de Rijk, Structural, optical and mechanical properties of coloured $\mathrm{TiN}_{\mathrm{x}} \mathrm{O}_{\mathrm{y}}$ thin films, Thin Solid Films 447-448 (2004) 449-454.

[15]F. Vaz, J. Ferreira, E. Ribeiro, L. Rebouta, S. Lanceros-Méndez, J.A. Mendes, E. Alves, Ph. Goudeau, J.P. Rivière, F. Ribeiro, I. Moutinho, K. Pischow, J. de Rijk, Influence of nitrogen content on the structural, mechanical and electrical properties of TiN thin films, Surf. Coat. Technol. 191 (2005) 317-323.

[16] I.C. Noyan, J.B. Cohen, Residual stress: measurement by diffraction and interpretation, Springer-Verlag, New York Inc., 1987. 